\title{
Error Source Analysis and Compensation Research of Laser Radar Scanning System
}

\author{
Peng Cang ${ }^{1,}$, Zhenglin $\mathrm{Yu}^{1}$ and Zheng Wang ${ }^{1}$ \\ ${ }^{1}$ Changchun University of Science and Technology, Changchun, 130022, China; \\ ayubo745@163.com
}

Keywords: Laser Radar; Error Source Analysis; Data Processing; Precision Compensation

\begin{abstract}
Aiming at the measurement characteristics of the laser radar scanning system, the relevant error source analysis is carried out, and the main factors influencing the measurement error are listed. At the same time, the influence of the error source on its data processing and measurement accuracy is discussed. And the corresponding compensation scheme and realization measures are proposed, which indicates the right direction of the realization of a more accurate measurement of the laser radar scanning system.
\end{abstract}

\section{Introduction}

With its high precision scanning, the corresponding speed, superior degree of automation and other characteristics, the laser radar scanning system are widely used in various measurement areas[1,2].The point cloud data acquired by the laser radar can be quickly generated to high-quality 3D model or digital point coordinates. However, in order to make better use of the equipment, it is necessary to consider the existence of the problem in practical applications. In the working of the laser radar scanning system, the laser radar is inevitable to be interfered by the external factors, which affects the accuracy of the measurement. Therefore, in order to make the acquired data more reliable, this paper analyzes and discusses the error sources of laser radar scanning system, the influence of error sources on the accuracy of point cloud data and how to reduce the error.

\section{Error Source Analysis of Laser Radar Scanning System}

The factors that cause data errors in the laser radar scanning system can be considered to be diversified $[3,4]$. For example: the divergence of the laser beam caused by scanning the process of positioning the angle deviation, the system installation and commissioning errors caused by external factors such as error. The error of the scanning system is transmitted according to the established rule of the measurement error. The laser radar scanning system is a multi-sensor fusion system, which drives the whole system through a certain axis linkage. Therefore, in the course of work, the error sources mainly include the following aspects:

(1) Deviations in the measured values of the angle and the distance due to the mutual rotation between the shafts.

(2) When the system works, the laser beam to the laser transmitter through the rotating mirror again at the object to be measured, the same high-speed rotating mirror will also measure the distance deviation.

(3) The calibration and calibration methods of the digital camera will have a certain deviation in measuring system.

(4) The errors caused by external factors, such as: weather, temperature, pressure, etc. will cause the deviation of the point cloud data and the measurement error caused by human factors.

(5) The surface smoothness of the measured object can also cause the deviation of point cloud data. For example, if the surface of the measured object is too smooth, it will cause multiple echoes at the same point on the surface, so that there will be some errors in the distance measurement . 


\section{The Analysis and Compensation of the Influence of Error Sources on the Precision of Point Cloud Data}

When the laser radar scanning system is used to scan the objects in the scene, the acquired information mainly includes point cloud data and image data. The description of the surface texture of the measured object can be obtained by simply processing the acquired image data according to the general photogrammetry processing method.

However, the point cloud data of the measured object is mainly used to express the accurate position information of the surface of the measured object, so the precision of the point cloud data is related to the deviation of the post processing 3D modeling and the real object. Therefore, it is very important to familiar with the influence of error sources and errors on the precision of point cloud data in point cloud data acquisition. This section focuses on the impact of random errors on the accuracy of point cloud data and the compensation method.

\section{The effect of Laser beam divergence.}

The core component of the laser radar scanning system is the laser scanner equipment used to acquire point cloud data. In general, the common methods of laser scanning ranging include: phase distance measurement, triangulation and pulse time ranging. At present, the mature laser range finder mainly uses the laser pulse time distance method to carry on the distance measurement. Laser pulse time ranging method is based on the time interval between the transmitted wave and the received wave to calculate the distance between the center of the scanner and the surface of the measured object. Due to the divergence characteristics of the laser beam, the size of the aperture that is irradiated to the surface of the object affects the accuracy and positioning effect of the retro-reflective point cloud. If it sets the laser beam to be circularly divergent, and the aperture to the surface of the object to be measured is expressed by Q0, the following formula is established.

$$
Q\left(\rho_{W}\right)=Q_{0} \sqrt{1+\left(\frac{\lambda \rho_{W}}{\pi Q_{0}^{2}}\right)^{2}}
$$

In the formula (1), $\rho \mathrm{w}$ is a distance parameter related to the position at which the laser beam is emitted. In general, the size of the aperture of the measured object is positively correlated with the measured distance, so the size of the aperture can be described by a linear equation related to the measurement distance. Currently, the laser rangefinder divergence aperture value will be given with the product together, for example, if Leica-HDS3000 aperture measurement distance is 50m, the aperture size is less than 6mm; if LMS-Z420i aperture measurement distance is $50 \mathrm{~m}$, the aperture size is13mm.

As the laser radar scanning system is to rely on the measurement of the center of the emitted beam measurement, and the divergence of the laser beam to the real point of laser ranging can't be determined, at the same time, before the measured object is measured, the shape and position of the measured object are also uncertain, therefore, therefore, the uncertainty of the beam must be quantified by a mathematical model before the measurement. The uncertainty of the laser beam width can be expressed by the following relation:

$$
P_{d}(\varphi, \gamma)= \begin{cases}\frac{4}{\pi \delta^{2}} & \gamma^{2}+\varphi^{2} \leq \frac{\varphi^{2}}{4} \\ 0 & \gamma^{2}+\varphi^{2}>\frac{\varphi^{2}}{4}\end{cases}
$$

In the equation(2), the parameter $\delta$ represents the beam diameter, $(\varphi, \gamma)$ represents the angle change when the beam is positioned. From the equation (2), the standard deviation of the beam can be solved. According to the characteristics of the beam density equation, the uncertainty of the position of the beam can be calculated by the empirical formula (3) as approximately $1 / 4$ of the diameter of the divergent aperture. 


$$
\sigma_{b}= \pm \sqrt{\int_{-\infty-\infty}^{+\infty} \int_{-\infty}^{\infty} \gamma^{2} P_{d}(\varphi, \gamma) d \varphi d \gamma}= \pm \frac{\delta}{4}
$$

In order to simplify the calculation, it is prescribed that the angular sampling interval $\varepsilon$ is the same between angle $\varphi$ and angle $\gamma$. The standard deviation expression (5) can be calculated and deduced from the density expression (4) to locate the beam center point:

$$
\begin{gathered}
P_{S}(\varphi, \gamma)=\left\{\begin{array}{cc}
\frac{1}{\varepsilon^{2}} & |\varphi|<\frac{\varepsilon}{2},|\gamma|<\frac{\varepsilon}{2} \\
0 & |\varphi|>\frac{\varepsilon}{2},|\gamma|>\frac{\varepsilon}{2}
\end{array}\right. \\
\sigma_{\mathrm{pc}}= \pm \sqrt{\int_{-\infty}^{+\infty} \int_{-\infty}^{+\infty} \varphi^{2} P_{S}(\varphi, \gamma) d \varphi d \gamma}= \pm \frac{\varepsilon}{2 \sqrt{3}}
\end{gathered}
$$

From the equation (5), it can be seen that the standard deviation of the beam center point is related to the sampling interval, that is, theoretically the same probabilities of the beam location center and the actual location center are related to the sampling interval.

Currently, most laser rangefinder positional deviations are only relevant for azimuthal measurements, with a small amount associated with positional deviation and also with pitch angle measurements. The influence of the observed noise and beam divergence makes the center of mass produce some uncertainties. Therefore, it is possible to reduce the deviation of the positioning by reducing the number of the centroid calculation points.

\section{The Impact of Coordinate System Conversion.}

The laser radar scanning system realizes the distance measurement of the surface of the measured object by automatic control of azimuth and pitch rotation. The coordinates of the point cloud that obtains the surface of the object in the system's custom coordinate system can be obtained by the formula (6), the measurement principle is shown in figure 1.

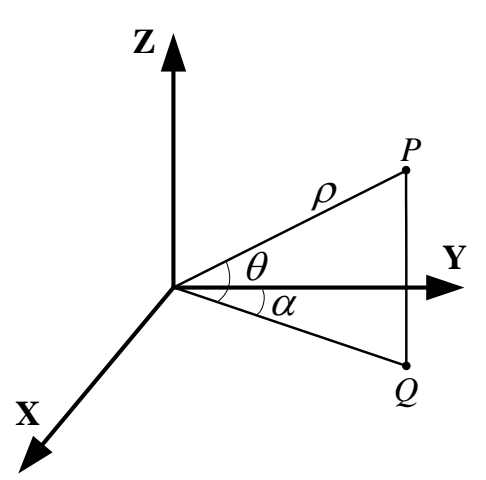

Fig.1 Schematic diagram of coordinate measurement

$$
\left\{\begin{array}{l}
x=\rho \cos \theta \cos \alpha \\
y=\rho \cos \theta \sin \alpha \\
z=\rho \sin \theta
\end{array}\right.
$$

In the equation (6), $\rho$ represents measurement distance, $\alpha$ represents an azimuth rotation angle, and $\theta$ represents a pitch rotation angle. This coordinate value is obtained in the custom scan measurement coordinate system. In order to facilitate the practical application, it is necessary to convert the point cloud coordinates in the scanning space coordinate system to the point cloud coordinates in the absolute coordinate system based on the earth coordinate system. The whole conversion relationship can be described by the equation (7). The whole conversion relationship diagram is shown in figure 2 . 


$$
\vec{r}_{t}=\vec{r}_{0}+R_{3}(k) \vec{r}_{S}
$$

In the formula, $\vec{r}_{t}$ is the coordinate vector of the measured object in the scanning space coordinate system, the formula is as follows:

$$
\begin{aligned}
& \vec{r}_{S}=\left(\begin{array}{lll}
x & y & Z
\end{array}\right)^{T}=\left(\begin{array}{lll}
\rho \cos \theta \cos \alpha \quad \rho \cos \theta \sin \alpha & \rho \sin \theta
\end{array}\right)^{T} \\
& \vec{r}_{t}=\left(\begin{array}{lll}
X & Y & Z
\end{array}\right)^{T} \text { is the measured object coordinates in the earth coordinate system vector. }
\end{aligned}
$$
However, $\vec{r}_{0}=\left(\begin{array}{lll}X_{0} & Y_{0} & Z_{0}\end{array}\right)^{T}$ is the coordinate vector of the station in the geodetic coordinate system, $\mathrm{k}$ is the horizontal angle between the measurement station and the rare viewpoint. The formula is:

$$
R_{3}(k)=\left[\begin{array}{ccc}
\cos k & \sin k & 0 \\
-\sin k & \cos k & 0 \\
0 & 0 & 1
\end{array}\right]
$$

To complete coordinate conversion, it is needed to determine the three conversion parameters (relative rotation matrix, the relative translation matrix and proportional value). For the laser radar scanning system, first of all, it is needed to determine the scanner coordinate system and the earth coordinate system Euler angle in order to determine the three conversion parameters. The size of the Euler angle determines what kind of coordinate conversion model algorithm people choose, because the different coordinate conversion model algorithm for the final conversion accuracy is different. Ceng Wenxian, Tao Benzao et al. proposed a non-linear transformation algorithm, which is suitable for the coordinate conversion system with small Euler angles. In the actual engineering survey, the Euler angle size is uncertain, therefore, in the coordinate conversion, it will not only encounter a small Euler angle, at the same time, will also encounter a large Euler angle. However, in the case of large angles, the conversion accuracy is poor if such a nonlinear coordinate conversion method is used. Li Yingcheng has made a study on the large Euler angles, and proposed a coordinate transformation model for large angle Euler angles. Recent studies have shown that, in addition to the model selection affects the final coordinate conversion accuracy, coordinate conversion accuracy is also related with the conversion of the reference point accuracy and the length of the baseline.

\section{The Impact of Station Installation and Rear Orientation Errors.}

In order to make the range finder work stable in the erection of laser radar scanning system, it is needed to make leveling and alignment operation for instrument, which inevitably introduces a certain system error, thus it will have an effect on the location and orientation of the measured object. In the course of laser radar work, azimuth error of scanning measurement is mainly affected by the location of the range finder and the orientation of the rear point. Assuming that the accuracy of the coordinate system in the horizontal direction is constant and the direction is constant, it can be written as $\sigma 1$. Since the observation points and the rear point errors are independent of each other, the relationship between the accuracy of the azimuth measurement and the horizontal distance can be obtained:

$$
\sigma_{\tau}= \pm \frac{\sqrt{2} \rho \sigma_{h}}{d_{0-B S}}
$$

The relationship between the accuracy of the horizontal angle measured by the scan and the horizontal distance is:

$$
\sigma_{\mu}= \pm \frac{\sqrt{2} \rho \sigma_{\varepsilon}}{d_{0-B S}}
$$

In the formula (11), $\sigma \varepsilon= \pm 0.5 \mathrm{~mm} / \mathrm{m}$ indicates the centering error, which is set according to the principle of alignment of the traditional instrument. 
The leveling effect of the range finder will have certain influence on the measurement accuracy of azimuth and pitch angle of the range finder.

The leveling effect of the range finder can be mapped by the rapid response characteristics of the leveling tube. The standard deviation of azimuth angle of the range finder is related to elevation angle deviation:

$$
\sigma_{l k}= \pm \sigma_{l v} \text { tan } \alpha
$$

The deviation formula of elevation angle is:

$$
\sigma_{I_{V}}= \pm 0.2 S_{n}
$$

In the equation (13), Sn represents the sensitivity of the level bubble. For devices with low sensitivity and no biaxial compensation system, the accuracy of the point cloud data is affected by the error significantly.

In view of the above analysis, it selects scanning measurement equipment according to the actual project requirements. If the conditions permit, in order to obtain high-precision scanning point cloud data, it will try its best to select the scanning measurement equipment with the level of better sensitivity or with biaxial compensation system, which can significantly reduce unnecessary scanning error.

\section{Conclusion}

(1) The main factors influencing the measurement accuracy of laser radar scanning system are analyzed. At the same time, the main reasons for its measurement error are discussed, which lays a solid foundation for realizing error compensation.

(2) The influence of the error source on the data processing and measurement accuracy of the laser radar scanning system is analyzed. At the same time, the specific measures to improve the accuracy of the acquired point cloud data are proposed. It provides a direction for achieving higher accuracy measurements.

\section{References}

[1] Xiang Zhiyu. Design and System Calibration of Fast 3-D Scanning Laser Radar [J]. Journal of Zhejiang University (Engineering Science), 2006, 40(12): 2130 2133.

[2] Chen Qiwei, Sun Gang, Pang Hewei, Tang Laiying, Yang Zaihua. Application of Laser Radar System in Large Scale Terrain Scanning [J]. Spacecraft Environmental Engineering, 2012, 29(2):215 219.

[3] Xia Jing, Jiang Lixing, Fan Xiaozhong. Airborne Laser Radar Positioning Error Analysis [J]. Journal of Geomatics Science and Technology, 2011, 28(5):365 369.

[4] Wang Chen, Li Chuanrong, Tang Lingli. Analysis and Correction of Airborne Laser Radar Data Error [J]. Remote Sensing, 2007, 11(5):390 397. 